\title{
Testing and calibration of smart pebble for river bed sediment transport monitoring
}

\author{
Ehad Akeila, Zoran Salcic \\ Department of Electrical and Computer Engineering \\ University of Auckland \\ Auckland, New Zealand
}

\author{
Nihal Kularatna ${ }^{1}$, Bruce Melville ${ }^{2}$,Ambuj Dwivedi ${ }^{3}$ \\ ${ }^{1}$ School of Science and Engineering, The University of \\ Waikato, ${ }^{2,3}$ Dept of Civil and Environmental Engineering \\ University of Auckland, New Zealand
}

\begin{abstract}
The Smart Pebble (smart particle), SP, has been developed for the past two years to monitor sediment transport in riverbeds. The implementation is based on use of small size and low cost acceleration and angular motion sensors. In this stage, the project is focused on calibrating and testing the final version of the SP as well as its packaging in a 4-cm diameter spherical package. The calibration was done in two stages; individual sensor calibration and complete system calibration. The complete SP unit was tested under linear motions generated by a shake table, and $2 D$ rotational motions using two manually controlled servomotors. Offline digital signal conditioning was done in MATLAB. The preliminary results show that the system has relatively large amplitude error due to low sampling frequency. Experiments conducted by sampling a 1-Hz sinusoidal signal at different rates show that to keep the amplitude error of the system under $5 \%$ the sampling rate has to be at least 10 times the maximum bandwidth of the signals acquired from sensors.
\end{abstract}

\section{INTRODUCTION}

Low cost micro-electro-mechanical systems (MEMS) for consumer and automotive applications have been also used in advanced engineering applications. References [1,2] indicate that low cost MEMS are usable in a strap-down inertial platform if the cumulative errors are managed with adequate accuracy. The success of a proof-of-concept project $[3,4]$, based on a mixed signal approach, proved recently that a "smart" sediment particle could provide significant insight into the process of sediment entrainment

The granular materials (sands and gravels) comprising the beds of rivers are typically in motion during periods of high river flow and stationary at other times. One of the biggest problems of these particles is that of the cause of the initial motion which lifts them up in water. This process is called "Entrainment or Pickup". Figure 1 depicts the test arrangement for use of the smart sediment particle in experiments designed to elucidate the sediment entrainment process. The smart particle will be positioned amongst a bed of similar fixed particles. Miniature load cells will be used to measure the inter-particle forces between the smart particle and its fixed neighbors.

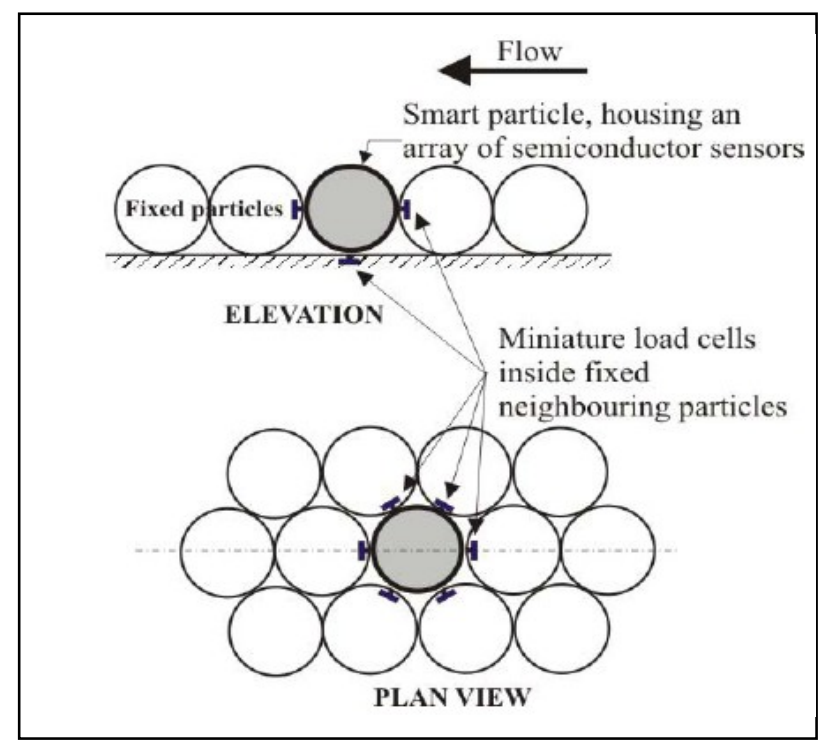

Figure 1: Test arrangement using the smart

In developing a "smart" pebble for this particular application, the following key features were essential in addition to the basic motion-related parameter: packaging, powering, relative physical placement of the MEMS, signal conditioning and providing a bare minimum digital subsystem and a compact and simple data communication interface. Packaging has direct influence on the size of the pebble. Since rough elements involved in the sediment entrainment study are $40 \mathrm{~mm}$ diameter ping pong balls, therefore the diameter of the pebble should not be more than $40 \mathrm{~mm}$ which is only possible with proper compact packaging of the embedded electronics. Entrainment is a sporadic and instantaneous event. The time at which particle will be entrained can vary from few seconds to few minutes. This directly put constraint on powering the SP for at least 15 minutes to capture the meaningful data before entrainment, during and after the pebble entrainment. Physical placement of the sensors and electronics should be such that the center of gravity is at the centre of the sphere (pebble) to avoid any abrupt motion due to eccentricity of the 
inside mass. Compact and simple data communication system is also required to make the pebble user-friendly

This paper is organized as follows: Section 2 describes the evolution of smart pebble since the first version in 2004. Section 3 describes the calibration process of the sensors and the SP system as a whole. The testing devices are shown in section 4 followed by the preliminary results in section 5 . Section 6 explains the effect of the sampling frequency and ends up with the conclusions in section 7.

\section{DEVELOPMENT STAGES}

The smart pebble project has evolved through several development stages since 2004. In each stage, further improvements have been made to achieve the project goals (packaging, accuracy, power consumption etc). These stages are:

1) 2004 summer project: This was the first work and it was required to prove the feasibility of the project and discover ways for monitoring the SP acceleration. It was discovered that the best way to achieve this is by using strap down Inertial Navigation System (INS). Such systems can determine their own position with respect to a fixed reference frame by means of measuring the accelerations and rotations about their frame. Euller's angles transformation is used to transform the accelerations from unfixed frame called Body Frame to a fixed frame called the Reference Frame [5]. According to this model, 6 sensors were used ( 3 accelerometer and 3 gyroscopes) and placed in the configuration shown in Figure 2. The equations and algorithms used in this stage can be found in [4].

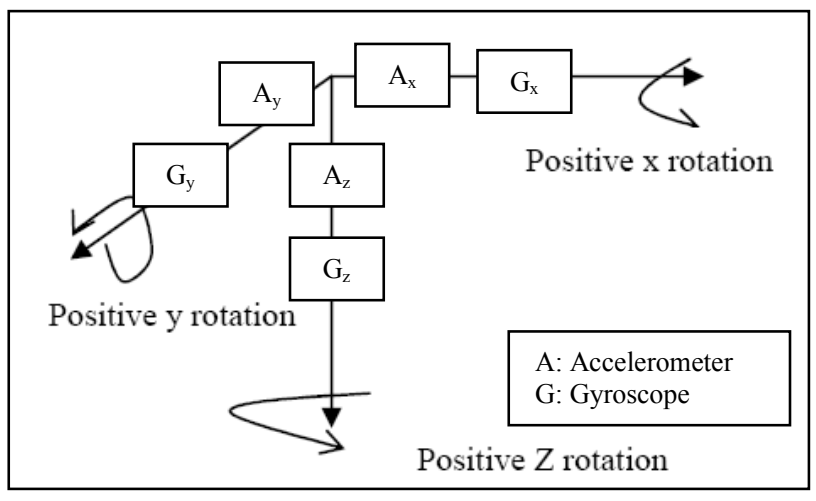

Figure 2: Axis conversion from body frame to reference frame.

2) 2005 project: The size of the SP was reduced using surface mount components. The power consumption was improved by replacing most of the analogue calculation circuits by a suitable microcontroller and a memory. At the end of this stage, the fisrt working prototype of the SP was made and packed into an 8-cm metallic ball as in Figure 3.

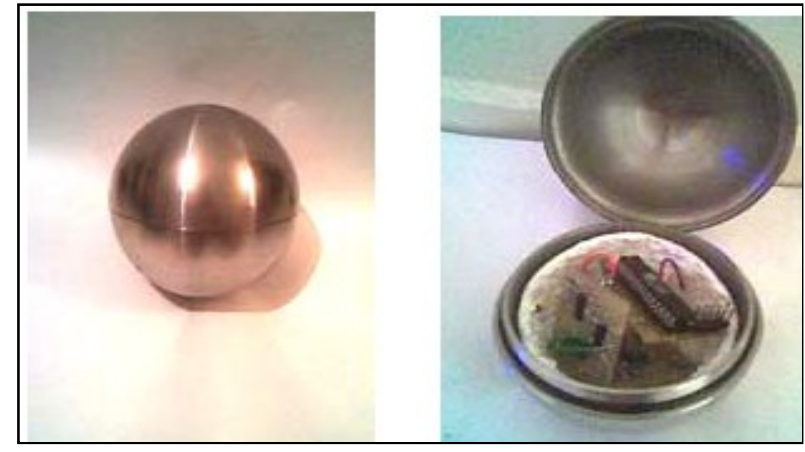

Figure 3: 2005 summer project smart pebble prototype.

3) 2006 project work: In this stage, the size of the PCBs has been reduced significantly. Before testing the SP, it was necessary to design a suitable package for all PCBs and the battery. Figure 4 shows the actual $4-\mathrm{cm}$ package with the PCBs inside.

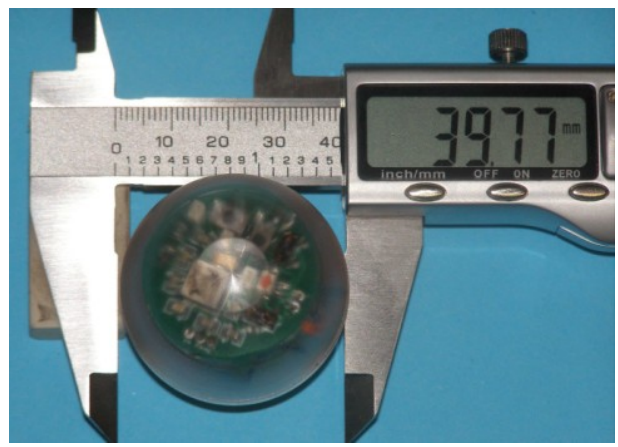

Figure 4: Smart pebble package

\section{CALIBRATION}

Calibration process was done in two independent steps: (1) individual sensor calibration (accelerometers and gyroscopes) and (2) calibration of the whole smart pebble system using MATLAB. Each of these steps is described in details in the following sections.

\section{A. Sensors calibration}

The accelerometer sensors (ADXL202) were calibrated against the gravity by orienting their sensing axes in the direction of gravitation to get $+\mathrm{g}$ output, and then in the opposite direction to get $-\mathrm{g}$ [6]. The gain and offset of the sensor are calculated using (1) and (2).

$$
\begin{aligned}
& \operatorname{off} \operatorname{set}(V)=\frac{V_{+g}+V_{-g}}{2} \\
& \operatorname{Gain}(V / g)=V_{+g}-\text { offset }
\end{aligned}
$$

Where $\mathrm{V}_{+\mathrm{g}}$ and $\mathrm{V}_{-\mathrm{g}}$ are the voltages at $+\mathrm{g}$ and $-\mathrm{g}$ respectively. 
The gyroscope sensors (ADXRS150) were calibrated by placing each sensor on a servo motor, which produces known angular velocities. The rotational speed of the motor was adjusted so that it does not exceed the gyroscope maximum sensitivity $(150 \% \mathrm{~s})$. This was done by controlling the voltage level that supplies the motor (at $3 \mathrm{~V}$ the speed is $83 \%$ s). Figure 5 shows the gyroscope calibration setup. The output of the gyroscope is shown in Figure 6

With reference to Figures 5 and 6, the gyroscope gives a high pulse $\left(\mathrm{V}_{+}\right)$when the motor rotates in a positive direction relative to the sensing axis $(+)$ of the sensor and a low pulse $\left(\mathrm{V}_{\text {-) }}\right.$ when moving in the opposite way. The sensitivity (gain) of the gyroscope is calculated using (3).

$$
\operatorname{Gain}\left(V /^{o} / s\right)=\frac{\left(V_{+}-V_{\text {offset }}\right) \Delta \mathrm{t}}{180}
$$

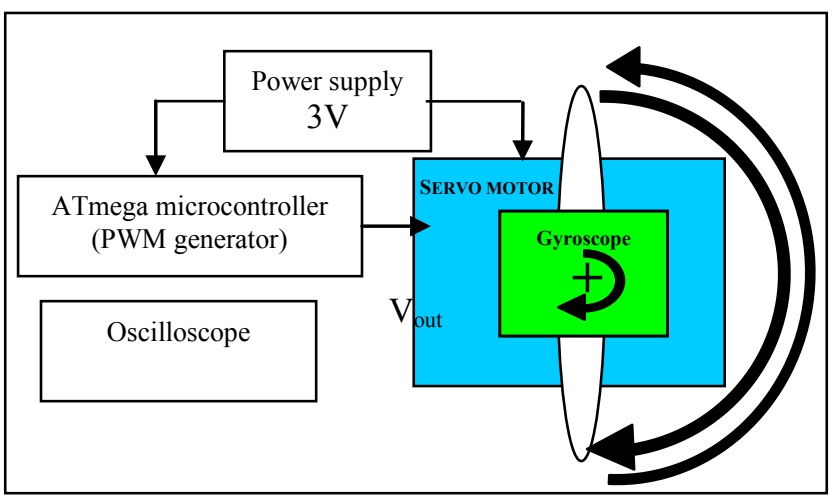

Figure 5: Gyroscope calibration setup

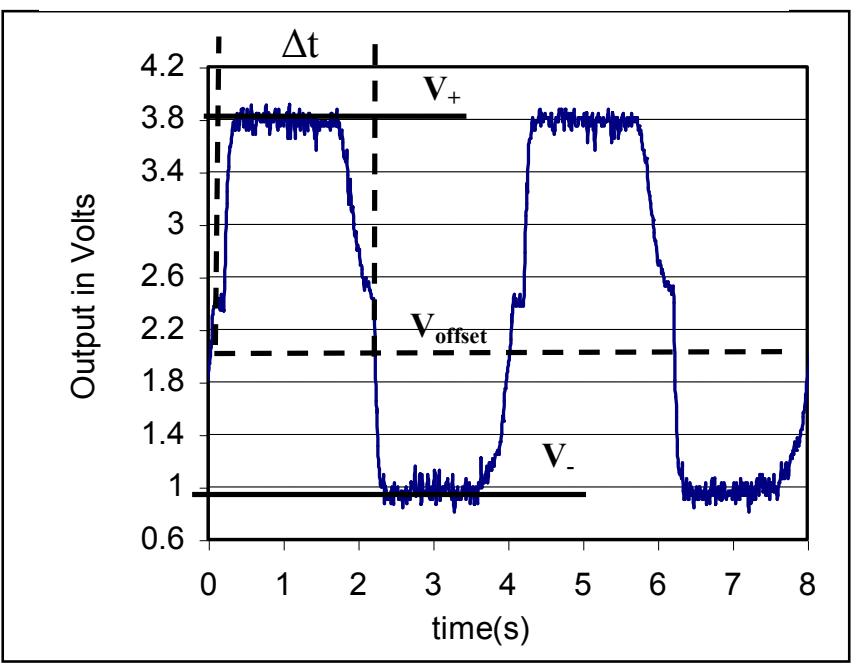

Figure 6: Gyroscope signal output that resulted from the servo motor rotation

\section{B. System Calibration}

The first step in this calibration process is testing the zero acceleration output (null offset) which is the output of the SP when it is left stationary. The total noise included in this version of the project has caused accumulation error when implementing digital integration on the outputs of the gyroscopes to get the final rotational angles. The null offset output was improved by filtering the data using some MATLAB built-in digital elliptic filters and then, taking the average of the first 5 seconds readings of each sensor output as reference for calculating the accelerations and angles.

Figure 7 shows a comparison between the filtered and unfiltered data when the SP was left stationary for about 15 minutes. According to the figure, the accumulating angle error of the 3 -axes is minimized after filtering.

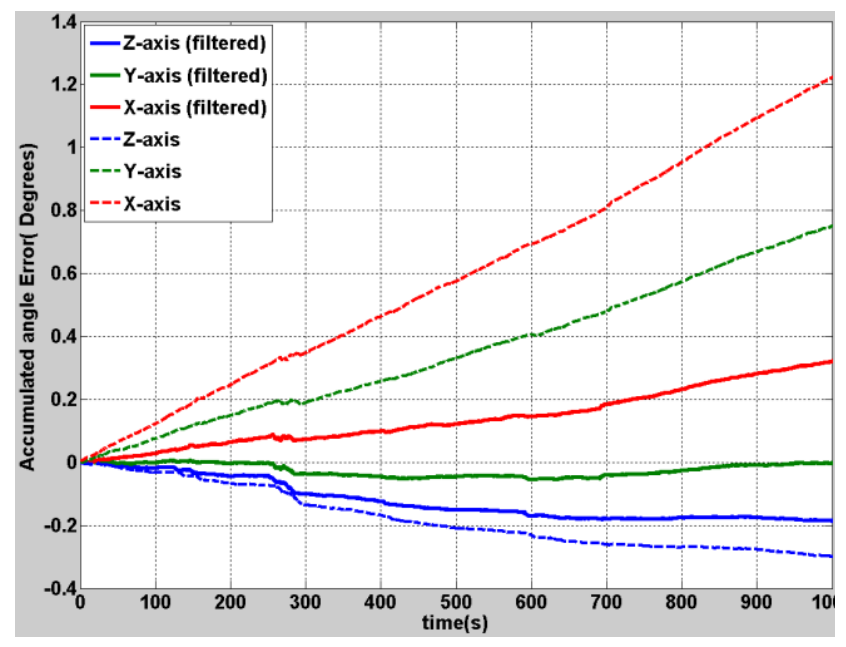

Figure 7: Accumulative angle error resulted from noise.

\section{Testing DeVICES}

In the real environment, the SP will be exposed to forces that make it move randomly inside water. These motions can be classified in two types: linear accelerations and rotational motions. The devices used to simulate each type of motions are:

a) Shake-Table: This device is used to simulate the effect of the earthquakes on structures and buildings. It has a highly sensetive single axis accelerometer that measures the accelerations generated by the table. The shake table was used in this project to simulate the linear accelerations on the SP system. Figure 8 shows a picture of the shake table used in the calibration process.

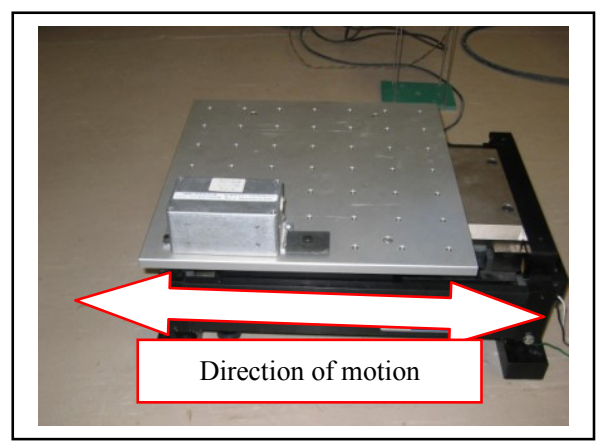

Figure 8: Shake Table 
b) $2 D$ rotational motors: This device was designed and built to generate rotational motions in two dimensions as shown in Figure 9. It is manually controlled by the user to rotate the SP at certain desired angles. The motors are then placed on the Shake table so that linear accelerations are combined with rotational motions at the same time. Figure 10 shows the final setup.

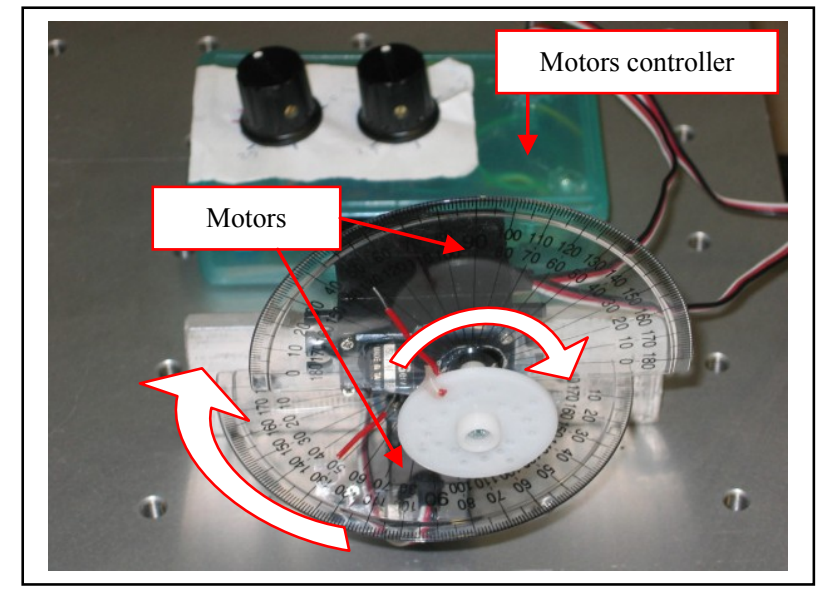

Figure 9: 2D rotational motors.

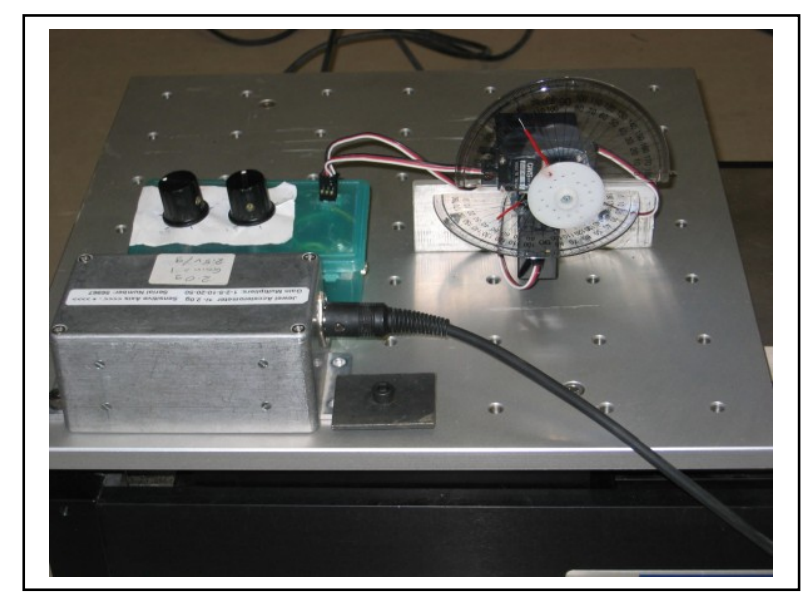

Figure 10: The complete SP testing devices setup

\section{PRELIMINARY RESULTS}

The Smart Pebble was tested first on the shake table by generating a $2 \mathrm{~Hz}$ sinusoidal moitions. Results show that the frequency response of the output is accurate enough to detect the frequency of the applied motion. Figure 11 is the frequency domain of the SP output showing a large peak at 2 $\mathrm{Hz}$ and some other frequency components occurring at the range of $1-2 \mathrm{~Hz}$ caused by aliasing.

However due to the noise and mainly the very low sampling frequency of the SP, the amplitude output error is very high (it ranges from $0 \%$ to more than $92 \%$ in some time intervals. Figure 12 shows a comparison between the SP output and the output from the shake table.

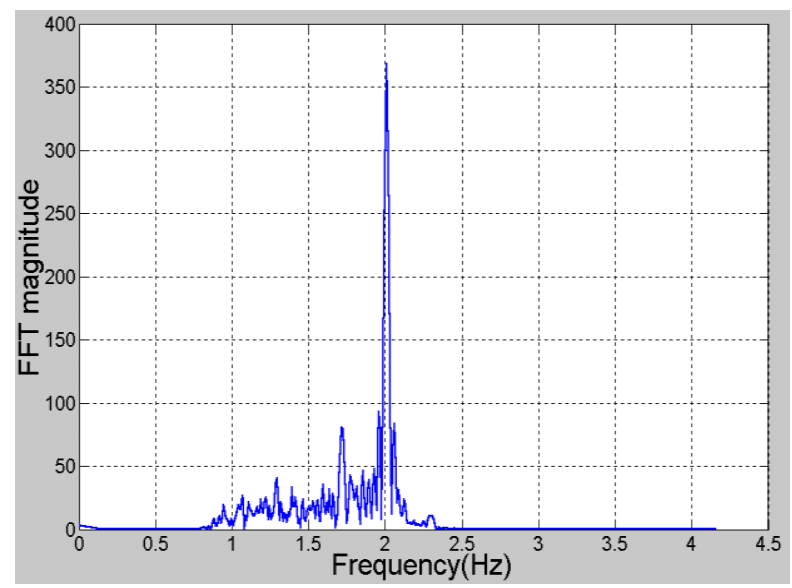

Figure 11: Power Spectral Density of the smart pebble x-axis

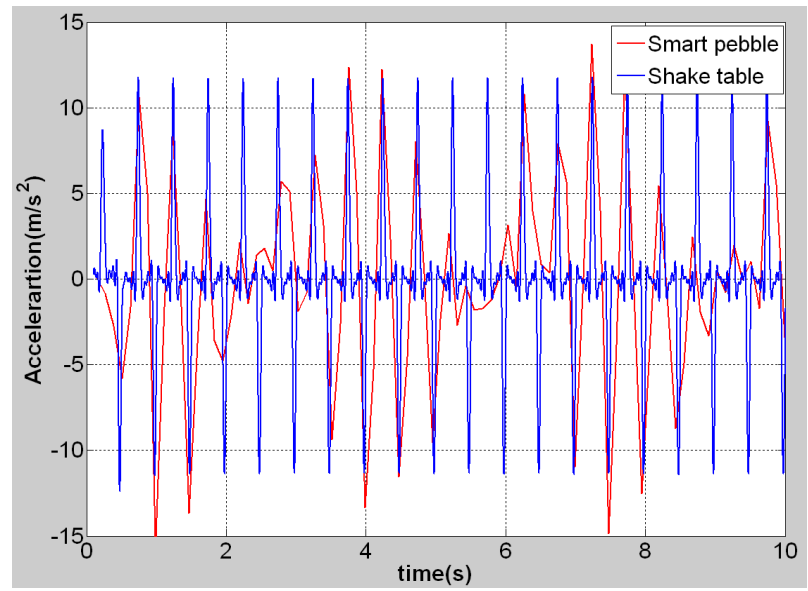

Figure 12: SP output versus the shake table output.

\section{EFFECT OF THE SAMPLING FREQUENCY ON THE ACCURACY}

Due to the large time-domain amplitude error, it was necessary to study the effect of the sampling frequency on the accuracy of the output amplitude. This was done by sampling a $1 \mathrm{~Hz}$ sine wave at different sampling rates using a 10-bit Analog to Digital Converter (ADC). The peak-to-peak amplitude of the sine wave has been adjusted to match the full scale range of the ADC analog as amplitude error $(\varepsilon)$ between the sampled ADC signal and the real signal is directly proportional to the ratio between the signal amplitude and the range of the ADC according to (4)[7]:

$$
\varepsilon=\frac{\sqrt{2} \pi V_{s_{P K}} B W}{\sqrt{5} f_{s} V_{F S_{P K}}}
$$


where $\mathrm{V}_{\mathrm{SPK}}$ is the input signal peak value, $\mathrm{V}_{\mathrm{FSPK}}$ : the ADC full scale range, $B W$ : Bandwidth of the analog signal, $\mathrm{f}_{\mathrm{s}}$ : Sampling frequency.

Figure 13 shows the graphs of the amplitude error percentage versus the ratio between the sampling frequency $\left(f_{s}\right)$ and the maximum signal BandWidth $(B W)$. According to Figure 13, the amplitude error drops significantly when the sampling frequency factor is in the range between 10-12 times of the maximum signal bandwidth, where the error is within 5\% as required in the Smart Pebble project.

According to Nyquist theorem, the sampling frequency should be at least twice the maximum frequency of the input signal in order to fully recover the signal. In our case, the sampling frequency of the system is $8.33 \mathrm{~Hz}$, which is about 4 times the frequency of the input. The error in the frequency domain is very small compared to it in the time domain. This implies that the factor of two in Nyquist theorem is required only to recover the signal in the frequency domain and avoid aliasing. However, this is not valid for time domain.

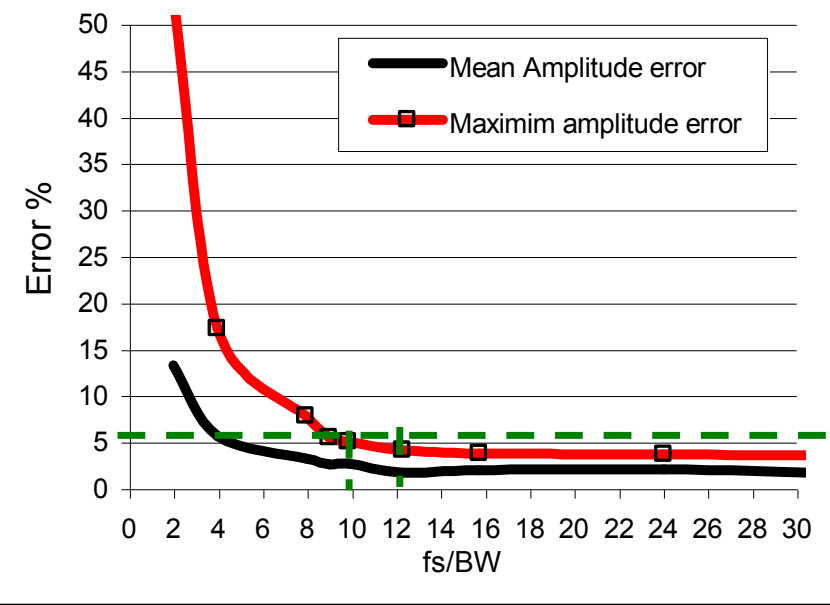

Figure 13: Amplitude percentage error at different sampling frequencies

\section{CONSIDERATIONS OF FUTURE IMPROVEMENTS}

We have explored future improvements of the smart pebble. The first thing considered was using a NonGyroscope Inertial Navigation System (NGINS), which will use only accelerometers for detecting the smart pebble motions. Using NGINS will have the advantages of minimizing the size of the PCBs in the first place as well as improving the accuracy of the system. This idea was explored using two and three 3 -axis accelerometers in $[8,9]$.

The other fundamental implementation issue is reducing the overall power consumption by selecting a very low power microcontroller, as the microcontroller in the current version of the smart pebble is consuming more than $80 \%$ of the overall power.

\section{CONCLUSIONS}

In conclusion, the smart pebble has been improved in its size and power consumption in the past three years. A 4-cm package has been designed and made in a way that fits the new PCB version and the battery. Calibrating the system was done in two independent steps: individual sensor calibration and overall system calibration. The initial system noise has been reduced offline by using digital filters which also reduced the accumulating angle error calculation from the gyroscopes. Shake table and 2D rotational motors were used to simulate the motions on the smart pebble.

Due to the low sampling rate of the current system, the time-domain amplitude error was too high. The minimum sampling rate required to sample any signal using an ADC is 10 times the maximum frequency component inside the signal, assuming that the peak of peak amplitude matches the full-scale range of the ADC component.

\section{ACKNOWLEDGMENT}

The authors would like to thank the help of the technical staff Geoff Kirby, Jim Luo and Mark Twiname of the civil engineering laboratories, in setting up the calibration system.

\section{REFERENCES}

[1] Barbour, N. \& Schmidt, G.: " Inertial sensor technology trends"; IEEE Sensors Journal, Vol. 1, No 4, Dec 2001, pp 332-339

[2] Brown, T.G et el; " Strap-down micromechanical (MEMS) sensors for high-G munition applications"; IEEE Trans on Magnetics, Vol. 37, No 1, Jan 2001.

[3] Kularatna, N. Melville, B. and Wijeratne, C. "Mixed Signal Approach for Rapid Prototyping of a Compact Smart Pebble for Sediment Transport Monitoring in River Beds", 2005 IEEE Sensors, Oct 2005, pp 1128 - 1132

[4] Kularatna, N. Melville, B. Akeila, E. Kularatna, D. “ Implementation Aspects and Offline Digital Signal Processing of a Smart Pebble for River Bed Sediment Transport Monitoring ", 2006 IEEE sensors, Oct 2007, pp 1093- 1098.

[5] Titerron, D.H. \& Weston, J.L.: " Strapdown inertial navigation technology"; IEE, 1997.

[6] "Calibration Techniques", Analog Devices technical article, Retrieved on July 15th 2006 from: http:/www.analog.com/Analog_Root/static/library/techArticles/ mems/Sensor971/sld59.html

[7] Patrick, H.G. "High performance instrumentation and automation". 2005

[8] D. Mingli, Z. Qingdong, and W. Qi, "The Application of Selfadaptive Kalman filter in NGIMU/GPS Integrated Navigation System," 2006.

[9] T. Chin-Woo and P. Sungsu, "Design of accelerometer-based inertial navigation systems," Instrumentation and Measurement, IEEE Transactions on, vol. 54, pp. 2520-2530, 2005. 